\title{
Tecnologías De Divulgación y Difusión Del Patrimonio Cultural y Natural Del Valle Del Mezquital, Hidalgo
}

DOI: $10.46932 / \mathrm{sfjdv2n2-172}$

Received in: March 1st, 2021

Accepted in: May 30th, 2021

\section{Cristy Elizabeth Aguilar Ojeda}

Instituto Tecnológico Superior del Occidente del Estado de Hidalgo, caguilar@itsoeh.edu.mx

Lorena Mendoza Guzmán

Instituto Tecnológico Superior del Occidente del Estado de Hidalgo,

Jorge Luis Rodríguez Ruíz

Héctor Daniel Hernández García, Luis Raúl Pérez Herrera

Colegio de México

\section{RESUMEN}

Algunos lugares culturales y naturales en el Valle del Mezquital, Hidalgo, son poco conocidos por parte de los habitantes de esta zona y de organismos gubernamentales; ello puede provocar la poca valoración y su conservación, sobre todo, una merma o tergiversación en las prácticas sociales y culturales que se realizan en torno a aquéllos. Por esta razón, se han desarrollado e implementado tecnologías que permiten la divulgación y difusión del patrimonio cultural y ambiental del Valle del Mezquital, con el objetivo de identificar, revalorar, reutilizar y promover este tipo de manifestaciones y/o lugares en la región, mediante la utilización de las Tecnologías de la Información (TI) que permiten la conformación de un repositorio web y a través de una aplicación móvil promoviendo su divulgación y difusión. Para lo cual se estructuró un marco teórico-referencial sobre herramientas de las TI y se consolidó un estado del arte sobre las mismas para tomar la decisión adecuada con respecto a las plataformas de desarrollo, usabilidad y viabilidad. Las TI han permitido que el conocimiento se difunda y divulgue a una mayor velocidad y con un mayor alcance, específicamente en términos de la divulgación del conocimiento científico, ya que se actualiza continuamente con información de investigadores, pero sobre todo de los mismos usuarios. Por tal motivo, la investigación está basada en una metodología inductiva que permite establecer las premisas del Centro de información del patrimonio cultural y natural a partir de cinco fases las cuales son: 1. Análisis y selección, 2. Investigación exploratoria, 3. Desarrollo interactivo de las herramientas del Centro de información, 4. Integración del Centro de información y 5. Finalmente la comprobación del mismo.

\section{INTRODUCCIÓN}

En la actualidad se tiene la idea de que el patrimonio en general es importante para fortalecer la identidad de la sociedad, ya sea mediante elementos arquitectónicos, escultóricos, paisajísticos o naturales; sin embargo, el conocimiento o la reflexión sobre las razones para considerarlos elementos patrimoniales por lo regular se dejan a los especialistas. Paradójicamente, la mayoría de las cartas Internacionales en favor de la salvaguarda del Patrimonio apuntan como aspecto fundamental la concientización de las personas o usuarios de esos bienes, es decir de la sociedad en general. Es este uno 
de los principales retos en la salvaguarda del Patrimonio, ya que se suele desconocer cómo preservar el patrimonio cultural y natural que no es reconocido como tal, y por tanto no se le valora.

La idea del patrimonio cultural y natural no es reciente, y tampoco lo es su difusión y su divulgación, ya que instituciones, ONG's, Organismos Mundiales se han dado a la tarea de realizar Simposios, Seminarios, Talleres, Foros, y otros eventos donde se discute sobre qué podría considerarse patrimonio cultural y natural, su protección, los lineamientos para su manejo, así como los involucrados. Sin embargo, estos eventos se realizan, generalmente, por y para personas interesadas y especializadas en el tema. Contrariamente a la intención y significado del concepto de patrimonio, la sociedad en general difícilmente tiene conocimiento de lo que ahí se discute; de los documentos en torno al patrimonio cultural y natural, incluso desconoce cuáles son los sitios considerados como tal; paradójicamente, la mayoría de los documentos refieren que es de vital importancia la participación de la sociedad en general, en tareas como la identificación y conservación de "su" patrimonio.

Ahora bien, ¿cómo identificar y/o reconocer los elementos que pueden ser considerados patrimonio ya sea cultural o natural?, se dilucidan dos maneras básicas, una es por un interés propio o social y la otra por redes de información y/o medios de comunicación, siendo este último el tema de este trabajo de investigación, tomando como referencia el de Desarrollo Interactivo e Incremental de Noriega; derivando en lo que comúnmente se conoce como divulgación y difusión. Aunque, quizá se tenga la conciencia de saber la importancia del Patrimonio por parte de la sociedad, en algunos casos, se desconocen las razones y las formas de preservarlo.

La importancia que tiene la divulgación y la difusión en la salvaguarda o conservación del patrimonio cultural y natural no es de ninguna manera reciente; han sido siglos de investigación y de disertación empírica. En décadas recientes las herramientas que coadyuvan a la difusión y divulgación del patrimonio por excelencia han sido y son las Tecnologías de la Información (TI). Éstas se han convertido en una herramienta vital para el quehacer humano, sobre todo por la velocidad en el traslado de la información, con medios como el internet, la telefonía celular, entre otros. Éstas, a su vez, han permitido que el conocimiento se difunda y divulgue a una mayor velocidad y con un mayor alcance. Las TI se vuelven una herramienta viable en la divulgación del conocimiento científico, al tiempo que resultan asequibles para informar sobre la importancia del patrimonio cultural y natural a la sociedad en general.

En términos del patrimonio cultural, tanto la Secretaría de Cultura (SC) [1] como la Secretaría de Turismo (SECTUR) [2] cuentan con sitios web en los que se aloja la información relacionada con la divulgación de algunos aspectos culturales y turísticos de los estados del país; sin embargo, no buscan difundir algún tipo de investigación científica a fondo sobre estos lugares, ya que sólo pretenden dar a 
conocer algunos datos relevantes sobre dichos lugares, sobre todo en el caso de la página de la Secretaría de Turismo.

Ahora bien, en el Estado de Hidalgo existen diversas asociaciones como el Consejo Consultivo Ciudadano de Pachuca, el Observatorio Ciudadano de Tula y el de Tulancingo, sólo por mencionar algunos. Los cuales cumplen con un papel restringido en la evaluación, propuesta y puesta en marcha de acciones en las áreas en las que se enfocan, debido a muchas veces a la dispersión de los esfuerzos, el desconocimiento, la falta de recursos o su afinidad con ciertos grupos políticos que acortan su rango de acción y sus posibilidades de autonomía. Con este trabajo se pretende dar una mayor difusión y divulgación de lugares, elementos o espacios que no son tan reconocidos en el Valle del Mezquital, incentivando el interés por estos, incluso activar dinámicas turísticas en su entorno.

\section{TEORÍA}

Proceso de identificación de los bienes culturales y naturales. La United Nations Educational, Scientific and Cultural Organization (UNESCO) en la Convención sobre la protección del Patrimonio Mundial, Cultural y Natural realizado en París en 1972, describe en su artículo 23 que “...prestará asistencia en los distintos momentos de la preservación del patrimonio, [ya sea la] Identificación; Protección, Conservación, Revalorización y Rehabilitación y evidentemente su Preservación” [3]. Dichos momentos sirven de base para entender el proceso de salvaguarda del patrimonio.

Con base en lo anterior, y para este trabajo, se describe el proceso metodológico para la salvaguarda del Patrimonio Cultural y Natural a partir del uso de la TI: 1. Identificación, Valoración, Valorización, 2. Difusión, Divulgación y Uso Sustentable, 3. Mantenimiento-Protección, 4. Conservación, 5. Revalorización, 6. Rehabilitación y 7. Preservación.

Los Centros de Información se ubican particularmente en el momento de identificación, valoración, valorización, es necesario entenderlos así de forma general y en lo tocante al presente trabajo.

Valoración. Como ya se mencionó, el primer paso que se tiene que hacer para la salvaguarda de un bien ya sea natural o cultural es la "identificación”, es el momento en que las personas reconocen algún valor a ese bien; Pérez identifica dos dimensiones de la valoración, una objetiva y otra subjetiva, la primera atravesada por las características del objeto mismo y la segunda por la subjetividad del sujeto [4], es decir, la valoración del patrimonio ya sea cultural o natural. Por lo regular los dos tipos de valores se dan de manera paralela y cuando se da esa "valoración", que puede ser de tipo social, cultural o ambiental, lo que resulta es el mantenimiento del bien. Sin embargo, suelen darse los casos donde se prioriza el aspecto objetivo, lo que puede provocar que la salvaguarda del bien tenga resultados ajenos a lo cultural, natural o social lo que implicaría un sesgo en un uso sustentable; entendiendo sustentable, ya sea natural o 
cultural, que debe generar el menor impacto al medio ambiente, ser económicamente rentable, pero sobre todo, tener un beneficio social amplio.

La identificación también está atravesada por procesos mentales, como la memoria, el significado y la reminiscencia los cuales “...coadyuvan a que la percepción y la relación que tienen las personas, de manera individual, con la obra arquitectónica, les permitan identificarse y reconocerse a través de la misma y apropiarse de ella de una manera única y personal" [5], aspectos que no deben desestimarse, ya que por lo regular son los que conforman la mayor parte de las tradiciones y costumbres, fortaleciendo la identificación y la apropiación de los bienes de tipo cultural o natural.

Centros de Información u observatorios. En los orígenes y las concreciones de la vida comunitaria es posible identificar a la dimensión natural y a la cultural como los dos grandes soportes de la vida en colectiva; debido a que, si bien deben ser entendidas como niveles distintos de la vida social y que ambos muestran determinaciones particulares que los vuelven niveles de definición práctica muy específicos, al mismo tiempo ambos se encuentran íntimamente relacionados y no pueden ser entendidos el uno sin el otro. Es así, que se propone una perspectiva que logre identificar y explorar esta relación de reciprocidad entre lo natural y lo cultural.

En este sentido, un observatorio o Centro de Información del patrimonio natural y cultural tiene como función principal ser un sitio de monitoreo de las condiciones de dichas dimensiones y lugares potencialmente considerados patrimonio, a partir de la generación de sinergias colectivas en las que se involucre a los actores sociales en el reconocimiento, cuidado, preservación y disfrute de los espacios. Estas sinergias posibilitan la articulación con actores fundamentales involucrados con la gestión de dicho patrimonio a partir de diferentes prácticas.

Si bien, estos Centros de Información cuentan con diferentes etapas durante su periodo de vida, es importante señalar que eso depende de las características que cada una de estas organizaciones y recursos con los que cuente. En el funcionamiento de los centros de Información pueden existir diversos tipos de agentes involucrados entre los que se encuentran el gobierno, la sociedad civil, la iniciativa privada, las Instituciones educativas, entre otros.

Patrimonio. En la Carta de Zacatecas escrita en XXIX Symposium Internacional de Conservación del Patrimonio Cultural organizado por ICOMOS Mexicano en la sección de las Conclusiones, se describe que "El Patrimonio no es sólo un conjunto de bienes y edificios con una relevancia cultural o histórica [o natural], sino la totalidad dinámica y viva de la creación del hombre y la naturaleza" [debido a que] "el patrimonio tiene un valor universal de proyección cultural e histórica que debe quedar reflejado en la adecuada respuesta de la sociedad a cada lugar" [6]. 
De manera general, se identifican dos tipos de patrimonios, el cultural y el natural; La United Nations Educational, Scientific and Cultural Organization (UNESCO) en la Convención sobre la protección del Patrimonio Mundial, Cultural y Natural realizado en Paris en 1972 caracteriza al Patrimonio Cultural [como] “...Las obras arquitectónicas, de escultura o de pintura monumentales, elementos o estructuras de carácter arqueológico, inscripciones, cavernas y grupos de elementos, que tengan un valor universal excepcional desde el punto de vista de la historia, del arte o de la ciencia, [asi como] los grupos de construcciones, aisladas o reunidas, cuya arquitectura, unidad e integración en el paisaje les dé un valor universal excepcional desde el punto de vista de la historia, del arte o de la ciencia y las obras del hombre u obras conjuntas del hombre y la naturaleza así como las zonas, incluidos los lugares arqueológicos que tengan un valor universal excepcional desde el punto de vista histórico, estético, etnológico o antropológico y el conjunto de bienes tangibles e intangibles, que constituyen la herencia de un grupo humano, que refuerzan emocionalmente su sentido de comunidad con una identidad propia y que son percibidos por otros como característicos”, mientras que Patrimonio Natural [como] “...los monumentos naturales constituidos por formaciones físicas y biológicas o por grupos de esas formaciones que tengan un valor universal excepcional desde el punto de vista estético o científico, las formaciones geológicas y fisiográficas y las zonas estrictamente delimitadas que constituyan el hábitat de especies, animal y vegetal, amenazadas, que tengan un valor universal excepcional desde el punto de vista estético o científico y los lugares naturales o las zonas naturales estrictamente delimitadas, que tengan un valor universal excepcional desde el punto de vista de la ciencia, de la conservación o de la belleza natural" [3].

Herramientas de Tecnologías de la Información. Antes de profundizar en el tema de las herramientas tecnológicas, es conveniente el principio en el que se basa la metodología y herramientas utilizadas con respecto a las TI en el presente trabajo. Dada la lógica del proceso de desarrollo de las versiones Alpha y Betha que más adelante se profundizará, se basan en Noriega el cual define al Desarrollo Interactivo e Incremental como: “...la construcción inicial de un pedazo de software, que va creciendo en forma gradual, ayudando a los involucrados en el proceso a descubrir lo más pronto posible problemas o inconformidades antes de que pueda llevar al desastre el proyecto" [7].

Una herramienta que puede ser usada para difundir y/o divulgar el Patrimonio Cultural y Natural que nos rodea, son las aplicaciones móviles, cuyo uso ha venido en aumento los últimos años. En este sentido una aplicación móvil o mejor conocida como APP, es un software diseñado para dispositivos como teléfonos o tabletas inteligentes [8]. Las aplicaciones móviles se han convertido en una de las principales formas en que la gente se comunica, compra, organiza sus vidas, juega e incluso trabaja.

Un estudio realizado en 2013 por la empresa iab México sobre usos y hábitos de dispositivos móviles, encontró que más del 80\% de las personas cuenta con un dispositivo móvil (teléfono celular, 
smartphone, tablet, videojuegos portátiles o mp3 con conexión a internet) y cuatro de cada diez usuarios cuentan con un teléfono inteligente o smartphone. Mientras que el promedio de descargas de aplicaciones es de 3 a 4 en un periodo de seis meses [8]

Ante tal panorama el desarrollar una aplicación móvil interactiva y de consulta, permitirá identificar los lugares de interés cultural y natural dentro del Valle del Mezquital.

MySQL workbench es un software que permite al usuario la posibilidad de modelado de datos, desarrollo de SQL y herramientas de administración completas para la configuración de servidores, administración de usuarios, copias de seguridad y mucho más, además permite diseñar visualmente, modelar, generar y administrar bases de datos. Incluye todo lo que necesita un modelador de datos para crear modelos complejos de ER, ingeniería directa e inversa y también ofrece características clave para realizar tareas difíciles de gestión de cambios y documentación que normalmente requieren mucho tiempo y esfuerzo [9].

- Desarrollado por: Oracle Corporation

- Programado en: C\#, C++ y Objective-C

- Sistema operativo: Microsoft Windows

- Licencia GNU General Public License

- Versión: 8.0.17

MySQL es un sistema de gestión de bases de datos relacional desarrollado bajo licencia dual: Licencia pública general/Licencia comercial por Oracle Corporation y está considerada como la base de datos de código abierto más popular del mundo, y una de las más populares en general junto a Oracle y Microsoft SQL Server, sobre todo para entornos de desarrollo web. Sea cual sea el entorno en el que va a utilizar MySQL, es importante monitorizar de antemano el rendimiento para detectar y corregir errores tanto de SQL como de programación [10].

- Desarrollado por: MySQL AB, Sun Microsystems y Oracle Corporation

- Programado en: C, C++

- Sistema operativo: Multiplataforma

- Versión: 8.0.17

Android Studio es una plataforma de Software basada en el núcleo de Linux. Fue diseñada en un principio para dispositivos móviles y permite controlar dispositivos por medio de bibliotecas desarrolladas o adaptados por Google mediante el lenguaje de programación Java. Todas las aplicaciones para Android se programan en lenguaje Java y son ejecutadas en una máquina virtual especialmente diseñada para esta 
plataforma, que ha sido bautizada con el nombre Dalvik. La licencia de distribución elegida para Android ha sido Apache 2.0, lo que lo convierte en software de libre distribución. Es un software que permite a los programadores crear aplicaciones para la plataforma, en la cual incluye proyectos, es un ejemplo de código fuente, herramientas de desarrollo, un emulador que incluye sus propias bibliotecas que se necesitan para poder construir la aplicación [11].

Con el SDK para Android, se pueden agregar mapas basados en datos de Google Maps a la aplicación. La API gestiona automáticamente el acceso a los servidores de Google Maps, la descarga de datos, la visualización del mapa y la respuesta a los gestos del mapa. También puede usar llamadas API para agregar marcadores, polígonos y superposiciones a un mapa básico, y para cambiar la vista del usuario de un área de mapa en particular. Estos objetos proporcionan información adicional para las ubicaciones del mapa y permiten la interacción del usuario con el mapa. La API le permite agregar estos gráficos a un mapa:

- Iconos anclados a posiciones específicas en el mapa (marcadores).

- Conjuntos de segmentos de línea (polilíneas).

- Segmentos cerrados (polígonos).

- Gráficos de mapa de bits anclados a posiciones específicas en el mapa (superposiciones de suelo).

- Conjuntos de imágenes que se muestran en la parte superior de los mosaicos del mapa base (superposiciones de mosaicos).

El editor de código de fuente Atom, de código abierto para macOS, Linux, y Windows con soporte para múltiples plug-in escritos en Node.js y control de versiones Git integrado, desarrollado por GitHub. Atom es una aplicación de escritorio construida utilizando tecnologías web [12].

- Escrito en: Electron

- Programado en: Electron (CoffeeScript / JavaScript / Less / HTML)

- Licencia: MIT License (free software)

- Modelo de desarrollo: código abierto

- Desarrollador(es): GitHub Inc

- Versión: 1.40.1

- Sitio web: https://atom.io/

Lenguajes de programación 
PHP: Hypertext Preprocessor (preprocesador de hipertexto), es un lenguaje de programación de propósito general de código del lado del servidor originalmente diseñado para el preprocesado de texto plano en UTF-8. Posteriormente se aplicó al desarrollo web de contenido dinámico [13].

- Desarrollado por: PHP Group

- Diseñado por: Rasmus Lerdorf

- Paradigma: Multiparadigma: imperativo, funcional, orientado a objetos, procedural, reflexivo.

- Versión: 7.2

JavaScript es un lenguaje de programación interpretado, dialecto del estándar ECMAScript. Se define como orientado a objetos, basado en prototipos, imperativo, débilmente tipado y dinámico. Se utiliza principalmente en su forma del lado del cliente, implementado como parte de un navegador web permitiendo mejoras en la interfaz de usuario y páginas web dinámicas [14].

- Desarrollado por: Brendan Eich

- Diseñado por: Netscape Communications Corp, Mozilla Fundation

- Paradigma: Multiparadigma, programación funcional, programación basada en prototipos, imperativo, interpretado

- Versión: 1.8.5

Tecnologías web: HTML es un lenguaje de marcado que se utiliza para el desarrollo de páginas de Internet. Se trata de las siglas que corresponden a HyperText Markup Language, es decir, Lenguaje de Marcas de Hipertexto [15].

- Tipo de formato: Lenguaje de marcado

- Tipo de MIME: text/html

- Versión: HTML5

CSS es un lenguaje de diseño gráfico para definir y crear la presentación de un documento estructurado escrito en un lenguaje de marcado.2 Es muy usado para establecer el diseño visual de los documentos web, e interfaces de usuario escritas en HTML o XHTML [15].

- Tipo de formato: Lenguaje de hoja de estilos

- Tipo de MIME text/css

- Versión: CSS3 


\section{PARTE EXPERIMENTAL}

\subsection{METODOLOGÍA}

Como ya se mencionó, para la conformación del Centro de Información se plantean cinco fases metodológicas, 1. Análisis y selección, 2. Investigación exploratoria, 3. Desarrollo interactivo de las herramientas del Centro de información, 4. Integración del Centro de información y 5. Finalmente la comprobación del mismo.

Sin embargo, es importante aclarar que para este trabajo se describen sólo lo correspondiente de la etapa uno a la etapa tres siendo esta última el resultado para este trabajo.

Fase 1. Análisis y selección. En esta fase de la investigación se realizó la recopilación de los sitios considerados con características patrimoniales, tanto de tipo natural como cultural. Dentro de ésta se categorizaron los lugares de acuerdo a cuatro subcategorías por cada una de las categorías generales, así también se realizó un conteo inicial de sitios que pueden ser dados a conocer a la población mediante las tecnologías de la información.

En lo referente a la elección de las TI más adecuadas en las cuales se basa la difusión, divulgación y el almacenamiento de la información dentro del Centro de Información, se desarrolla la metodología scrum como estrategia de buenas prácticas para el desarrollo óptimo de la investigación, la cual consta de cinco procesos que cubren una secuencia lógica de diseño y funcionamiento. Además, con el fin de entender completamente cómo las personas interactúan con la app, se analizaron tanto las interacciones directas del usuario con el dispositivo y la aplicación, así como el contexto externo que éste percibe durante su interacción.

Esta fase se encuentra estructurada en cinco sub-fases [16], las cuales se sintetizan y se describen los resultados a continuación.

1.- Diseño de instrumento de recolección de datos. En esta primera etapa se generó una encuesta que incluía preguntas para identificar los lugares que puedan ser susceptibles para considerarse como patrimonio cultural y/o natural, así como la frecuencia con la que son visitados. En este sentido, los resultados fueron: en el caso del patrimonio cultural se destaca que la Zona Arqueológica de Tula y el Ex Convento de Actopan son los lugares más reconocidos y con mayor número de ocurrencias, resultando 204 y 37 respectivamente de 400 encuestados, en un tercer lugar aparece la escultura de la Diana Cazadora de Ixmiquilpan con 31 ocurrencias, en cuarto lugar la pirámide de Taxhuadá con 28, en quinto el Puente de Tunititlán con 13, en sexto el Centro Cultural del Valle del Mezquital con 12 ocurrencias y en séptimo el Ex Convento de San Miguel Arcángel con 9. Para el caso del Patrimonio Natural se mencionan lugares como las Grutas de Tolantongo o la serie de Balnearios del corredor de Ixmiquilpan, los cuales fueron los más nombrados con 82 y 13 menciones respectivamente, si bien estos sitios pueden, desde luego, ser 
considerados en cierta medida como espacios con características naturales destacables. Sin embargo, nuestra atención se enfoca en aquellos lugares con menos ocurrencias.

2.- Cálculo de la muestra. A partir de la ecuación para calcular la muestra con base en Sampieri [17], se obtuvo una muestra de 383.48 encuestados, considerando un nivel de confianza del $95 \%$ y un margen de error del 5\%; a partir de la referencia de 216, 097 hogares que hay en el Valle del Mezquital.

3.- Aplicación del instrumento. Se aplicaron 400 encuestas (de septiembre a noviembre de 2016) a personas entre 13 y 79 años de la región del Valle del Mezquital, Hidalgo, de manera personal con base en el instrumento presentado anteriormente; y a través de la plataforma e-encuestas se analizaron los resultados, dicha encuesta se puede consultar en el siguiente link. http://www.eencuesta.com/answer?testId=AiCY9u658Dg= . De las 400 encuestas aplicadas, 53\% correspondió al sexo femenino y $47 \%$ al sexo masculino, se destacó el hecho de que el $71 \%$ de los encuestados no conoce el concepto de patrimonio cultural y natural, sin embargo, logra identificar lugares con reconocimiento social o local.

4.- Análisis de resultados. Para analizar los datos se utilizaron las herramientas proporcionadas por e-encuestas y hojas de cálculo, donde se cruzaron datos como edad, sexo, lugar de residencia, escolaridad, con el nivel de conocimiento sobre los conceptos de patrimonio cultural y natural, lugares que las personas del Valle del Mezquital identifican en estos términos y la posibilidad de utilizar herramientas tecnológicas para la difusión y divulgación de los lugares identificados. Ahora bien, en la encuesta también se preguntó sobre aquellos Instrumentos tecnológicos de difusión y divulgación del Patrimonio y el resultado fue que el teléfono celular es la herramienta tecnológica más utilizada sobre la misma computadora. La pertinencia sobre esta herramienta se puede observar que, al $87 \%$ de los encuestados les sería útil una herramienta que le brinde información sobre espacios de patrimonio natural y cultural; por otra parte, el $88 \%$ cuenta con un teléfono móvil inteligente y el sistema operativo que predomina corresponde a Android con un $82 \%$.

5.- Categorización de sitios naturales y culturales. Para ordenar los datos obtenidos a partir de las encuestas y la investigación de campo sobre los sitios con valor patrimonial natural existentes en la región, se propone dentro de este proyecto la clasificación desde una visión de los llamados servicios ambientales, que responde a una decisión más bien pragmática y se inscribe únicamente dentro de un contexto de utilitariedad conceptual, mientras para la clasificación de los sitios o elementos culturales se toma en cuenta principalmente la época de su construcción. Como resultado se establecieron, para el patrimonio cultural, se retomaron las categorías históricas que establece Enrique de Anda [18]. La prehispánica antes de 1498; Época Virreinal (1492-1810), Época posindependentista-revolucionaria (1810-1910), Época Moderna: se identifica a partir de 1910 hasta nuestros días. En lo que se refiere a los lugares naturales que 
pueden ser considerados como patrimonio natural se establecen las siguientes subcategorías con base en lo descrito por Onaindia: Servicios de Soporte, Servicio de provisión, Servicios de regulación, Servicios simbólicos y Ambiental [19].

\section{Fase 2. Investigación exploratoria}

Análisis de la usabilidad de las tecnologías de la información. Formalmente, la definición más utilizada o reconocida de usabilidad es la que se expone en la norma ISO 9241-11 en la cual usabilidad se describe como el grado con el que un producto puede ser usado por usuarios específicos para alcanzar objetivos específicos con efectividad, eficiencia y satisfacción, en un contexto de uso específico [20]. La norma define cómo especificar y medir la usabilidad de productos y aquellos factores que tienen un efecto en la misma; también destaca que la usabilidad en terminales con pantalla de visualización es dependiente del contexto de uso y que el nivel de usabilidad alcanzado dependerá de las circunstancias específicas en las que se utiliza el producto. El contexto de uso lo forman los usuarios, las tareas a realizar, el equipamiento (hardware, software y materiales), así como también los entornos físicos y sociales que pueden influir en la facilidad de uso de un producto.

En el campo de las TI, Solano (2015) [21], asegura que las personas interactúan entre ellas y con diversos dispositivos tanto de hardware como de software para llevar a cabo una serie de tareas. Los sistemas que actúan de interconexión entre las personas, y que favorecen la realización de las tareas y el alcance de los objetivos propuestos, son los denominados Sistemas Interactivos Software.

El desarrollo de software pasó por una serie de etapas desde su creación, a un nivel diferente de depuración, por lo que en el estudio Alpha, el grado de satisfacción de los usuarios, mostró que el 93\% de los encuestados describen que tienen un grado de satisfacción bueno (87\%) y un número muy bajo (6\%) tienen un grado de satisfacción no adecuado, sin duda, este último universo nos permitirá perfeccionar la interfaz y el contenido de la aplicación. Con base en lo anterior, la gran mayoría de los encuestados estaría de acuerdo en descargar la aplicación (87\%) ya sea por su objetivo, su interfaz o su contenido, como antes se mencionó, esto nos describe que la aplicación está siendo bien aceptada por las personas y que estarían de acuerdo en continuar utilizándose.

Un aspecto fundamental para poner en funcionamiento la aplicación móvil es pasar de la versión Alpha a la Betha, por lo que es necesario que sea evaluada la primera con usuarios piloto y posteriormente por los usuarios finales; esto para determinar aspectos como la interfaz, el contenido, la estructura y la apariencia además de la usabilidad que es considerada uno de los factores más importantes dentro de la calidad de un producto de software. 
Para conformar la versión Betha y mejorar la experiencia del usuario, se atendieron los factores tanto internos como externos del sitio web y de la aplicación móvil; por lo que la usabilidad se evaluó con base al alcance y la flexibilidad de sus funciones, tomando en cuenta la experiencia del usuario y con la identificación de problemas específicos, en términos de la apariencia del análisis anterior, el $48 \%$ de los encuestados refirió que es buena, esto nos describe que la versión Alpha no tuvo cambios mayores, sin embargo, hubo un $26 \%$ de encuestados que consideró que es regular, que habría que hacer algunos ajustes (colores, menú, tipografía). En este sentido, se buscó que la interfaz de la aplicación fuera lo más sencilla posible de utilizar, sin muchos cuadros de diálogo para evitar confusión y sobre todo que consuma un mínimo de datos.

Por lo que a continuación se muestra el desarrollo realizado para complementar y mejorar la apariencia y usabilidad de la versión definitiva.

Diagrama de interacción. Inicialmente se realizó el diseño del diagrama de interacción, que permite una visualización completa de la funcionalidad de las tecnologías, así mismo destacar las características principales del sitio web y la aplicación móvil. Posteriormente la base de datos permite la administración de cada uno de los datos de los diferentes usuarios así como de la información de los lugares y de la implementación de medidas de seguridad para evitar algún suceso ilícito. Los módulos que se modificaron fueron:

- Inicio de sesión.

- Registro de usuarios.

- Registro y administración de lugares.

- Localización de lugares en mapa de Google Maps.

Figura 1 Diagrama de interacción TI

API Google Maps

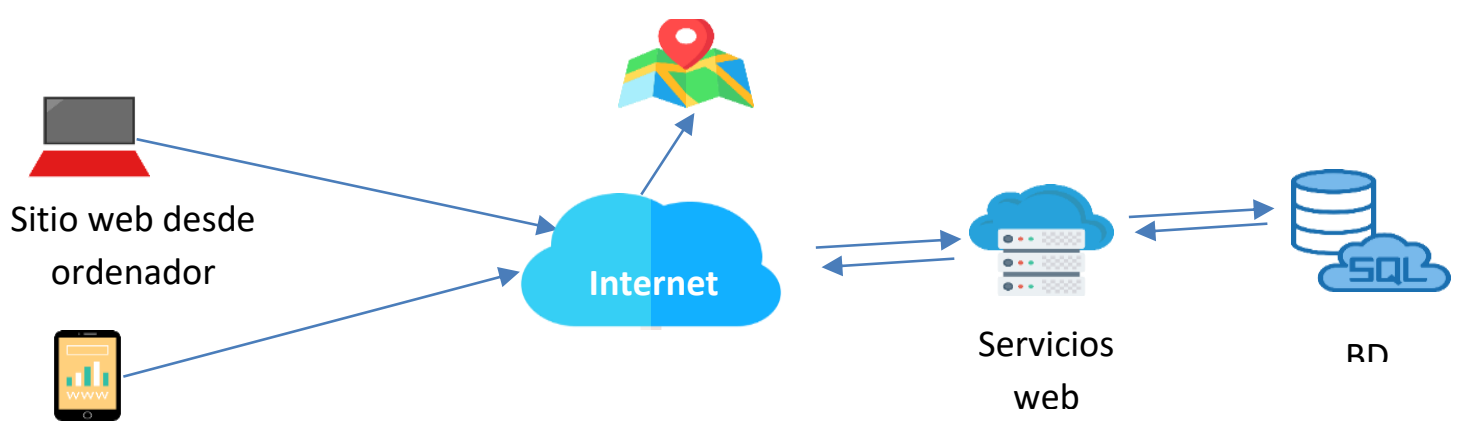

Aplicación

móvil 


\section{RESULTADOS}

Derivado de la importancia del reconocimiento de los lugares culturales y naturales así como de los resultados presentados en la caja de pruebas funcionales y de usabilidad a la versión final de las tecnologías, a continuación se presentan los resultados de la fase 3. Desarrollo interactivo de las herramientas del Centro de información, donde se definen las TI para el Centro de Información del Patrimonio Cultural y Natural del Valle del Mezquital.

En la pantalla de inicio del sitio web (Figura 2) se muestra la interfaz principal de los usuarios, donde se puede visualizar de fondo, imágenes de los paisajes que se encuentran en el Valle del Mezquital, en segunda instancia se encuentran los accesos a las diferentes secciones de la página. Entre estos se encuentran:

Figura 2. Pantalla principal del Sitio Web

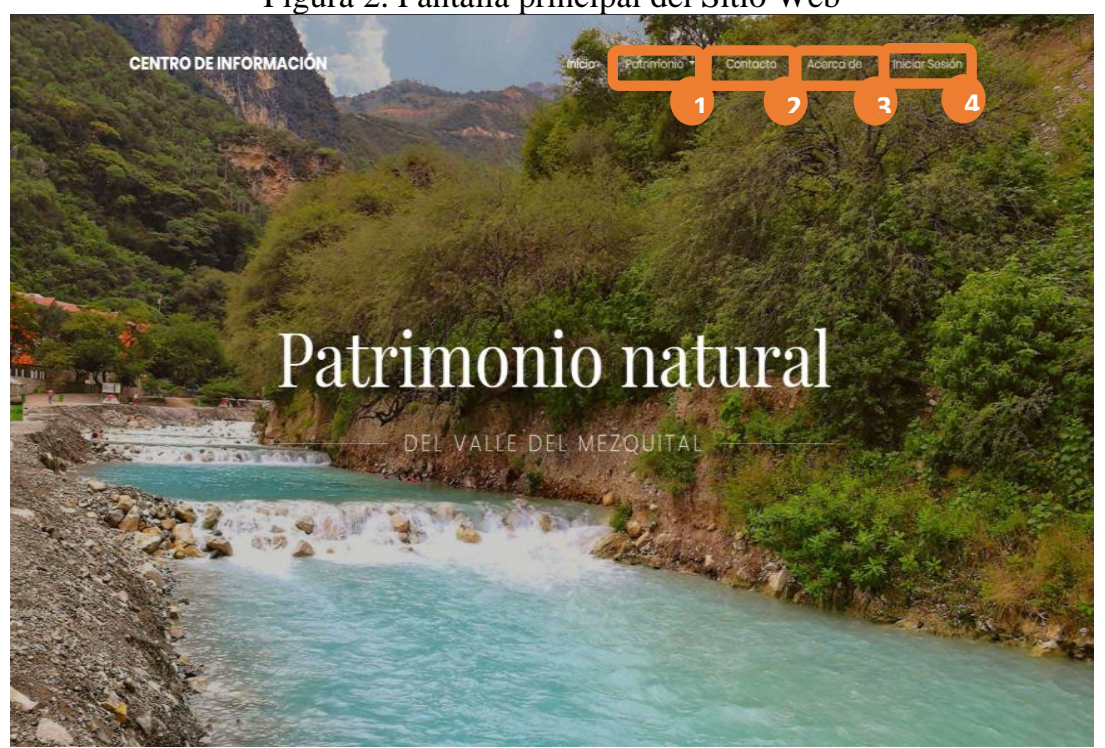

Patrimonio: despliega un menú con opciones de información Contacto Acerca de Iniciar Sesión

Al deslizar a la parte inferior de la pantalla principal, se encuentra la reseña del patrimonio cultural y natural del Valle del Mezquital y una galería de imágenes como se puede apreciar en la Figura 3. 
Figura 3 Reseña del Centro de Información

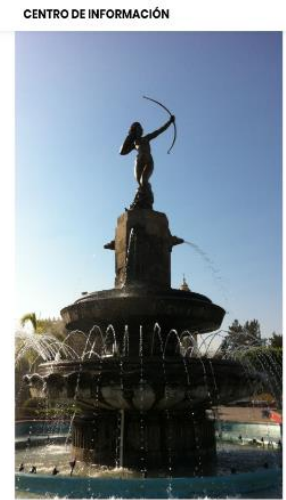

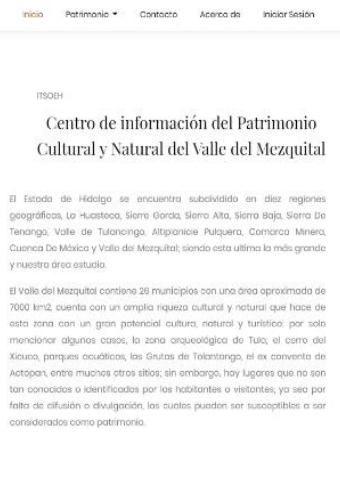

Figura 4 Menú Patrimonio Natural
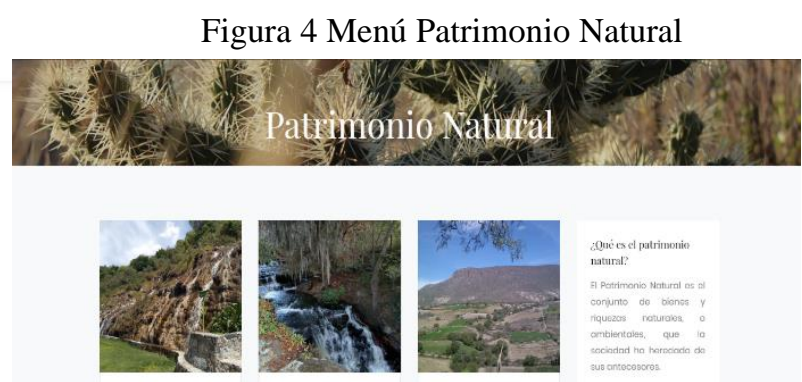

cerro del Elefefante
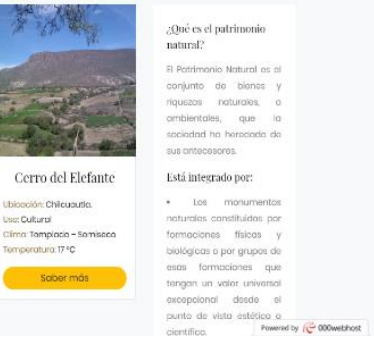

Como se puede observar en la Figura 4 y 5, tanto en el menú del "Patrimonio Natural" como de "Patrimonio Cultural" se pueden visualizar los lugares naturales y culturales que se encuentran en el Valle del Mezquital, además de los conceptos que lo integran.

El botón de "Saber más", que se puede apreciar en la Figura 5, dirige a otra pantalla la cual muestra la información del lugar seleccionado, así como sus características, la localización en el mapa, además del extenso que contiene la descripción completa del lugar (Figura 6).

Figura 5 Menú Patrimonio Cultural Figura

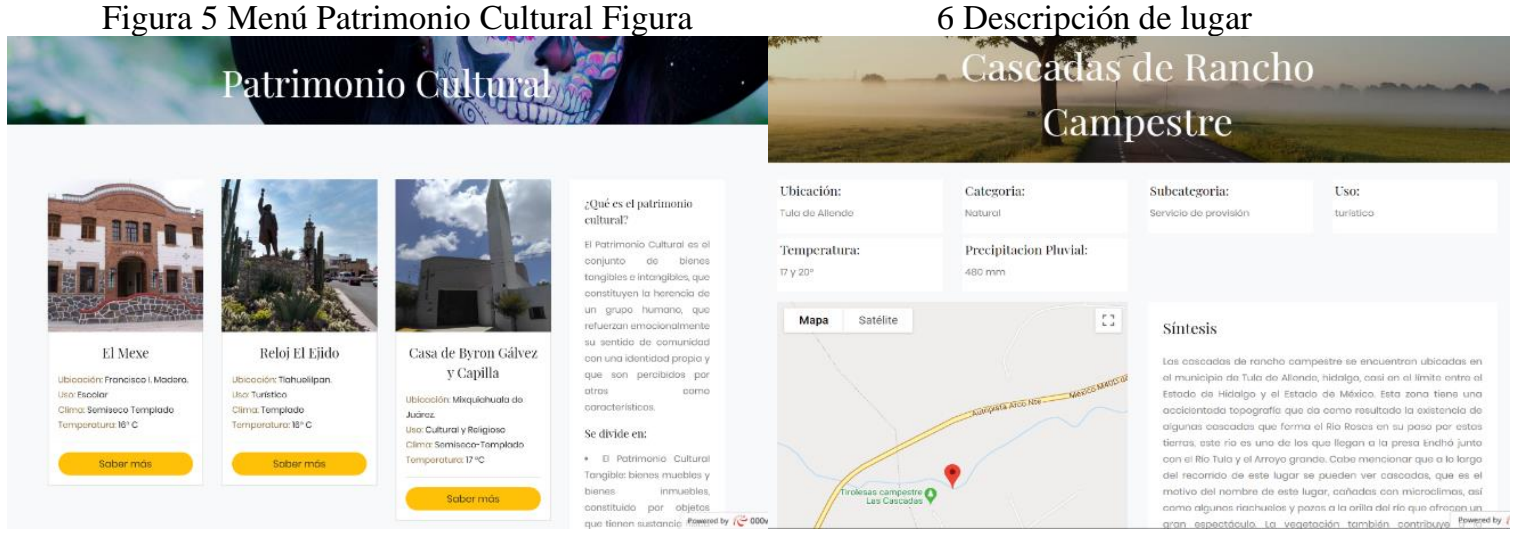

Además, con la finalidad de mantener una relación con el usuario final, se ha desarrollado el apartado de "Contacto" (Figura 7), para recolectar información que contribuya en la mejora continua de los datos publicados y de las mejoras que a criterio de los usuarios es necesario al momento de navegar en el sitio. Así mismo, se puede apreciar en la Figura 8, el menú "Acerca de", que muestra de manera detallada el propósito general del sitio web. 


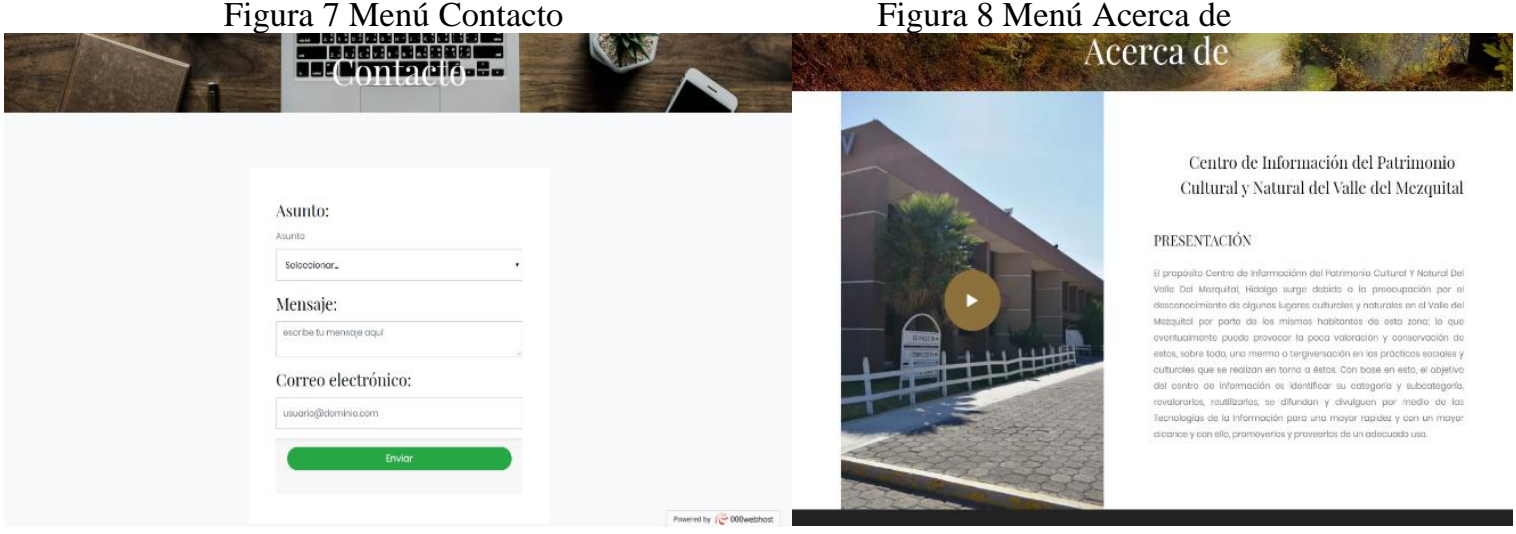

Finalmente, para que los administradores tengan la posibilidad de documentar los nuevos lugares analizados y de compartir datos relevantes que se considera de interés del usuario final, el sitio web tienen habilitada una sesión para actualizar la información del sitio web (Figura 9).

Figura 9 Acceso Inicio de Sesión
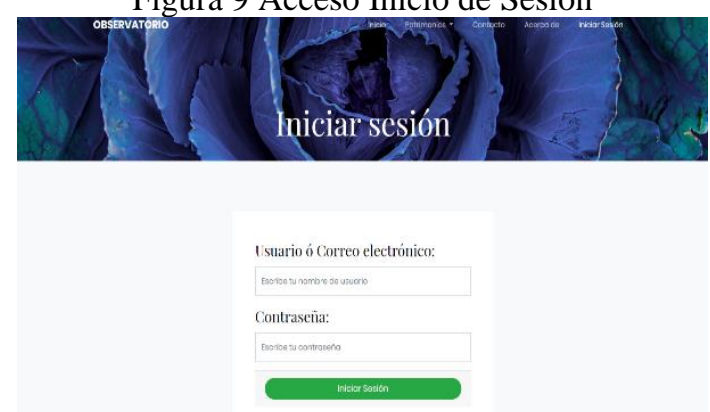

¿Aun no tienes una cuenta? Revistratcanui

En cuanto a la interfaz de usuario de la aplicación móvil, en la pantalla principal se muestra el inicio de sesión, para los usuarios registrados, y una opción de registro. Una vez ingresado correctamente con el usuario registrado, mostrará el menú de inicio. El menú de inicio (Figura 11) muestra cuatro botones, en los cuales se encuentran:

1. Patrimonio Natural y cultural.

2. Lugares naturales.

3. Lugares culturales.

4. Acerca de.

Estas opciones permitirán acceder a la información y navegar en la aplicación. 
Figura 10 Pantalla acceso aplicación móvil

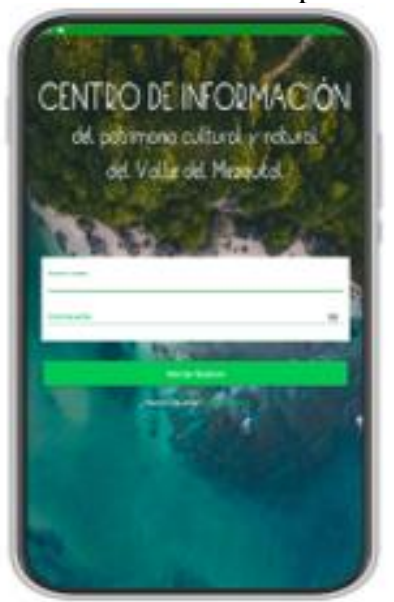

Figura 11 Menú de inicio

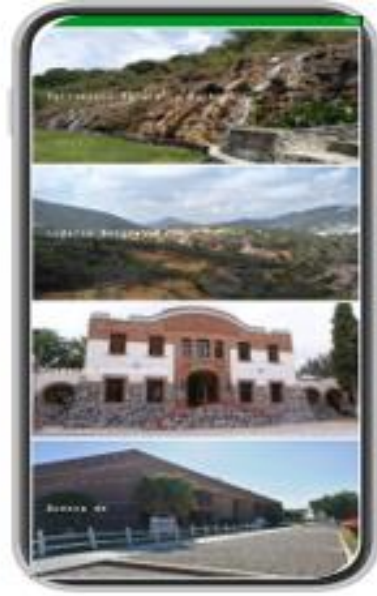

Figura 12 Descripción del Patrimonio

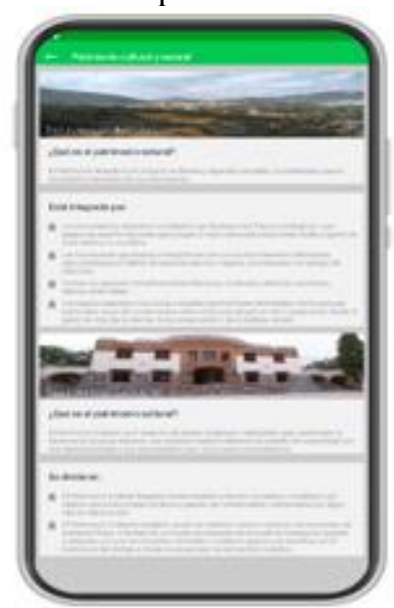

En la Figura 12, se muestran conceptos generales detallados del patrimonio cultural y el patrimonio natural. Al seleccionar la opción de Lugares Naturales (Figura 13-14), se muestra un mapa de geolocalización, en el cual se identifica con el marcador, el lugar seleccionado por el área de "selecciona un lugar natural" y un botón "ver información". Al abrir la lista desplegable del área "Selecciona un lugar natural", se muestran los lugares naturales disponibles, posicionando dicho marcador en el mapa, la localización del lugar que se seleccionó.

Una vez seleccionada la ubicación, con ayuda de las herramientas de Google Maps, se puede marcar la ruta de destino, pulsando sobre los botones: 1 . Cómo llegar y 2 .

Figura 13 Acceso lugares

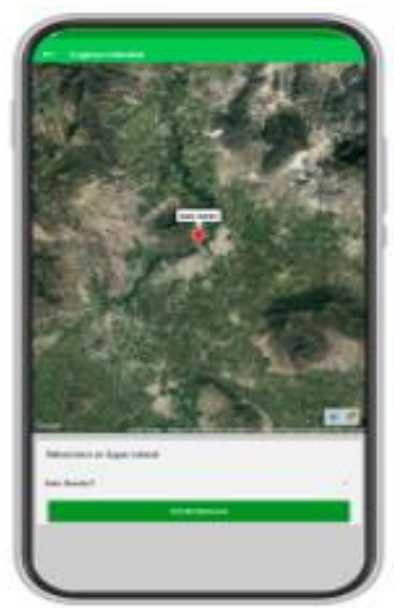

Figura 14 Ruta de lugares

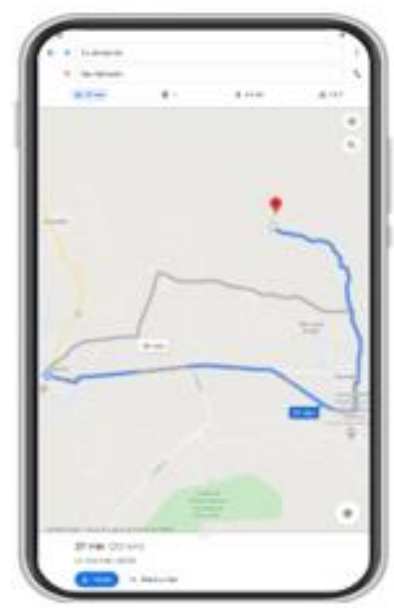

Una vez seleccionada la localización deseada, al presionar sobe el botón "Ver información”, abrirá una nueva pantalla en la que se podrá visualizar información del lugar seleccionado como la localidad donde se encuentra, clima, temperatura, precipitación pluvial, y un pequeño resumen de lo que es y se encuentra en este lugar, como puede observarse en la Figura 15. 
En el apartado "Acerca de (Figura16), se encontrará información sobre el fin que tiene la aplicación del Centro de Información”.

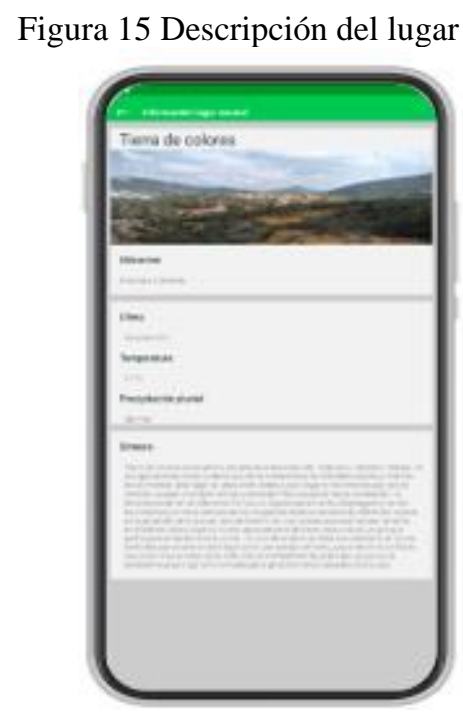

Figura 16 Descripción del Centro de Información

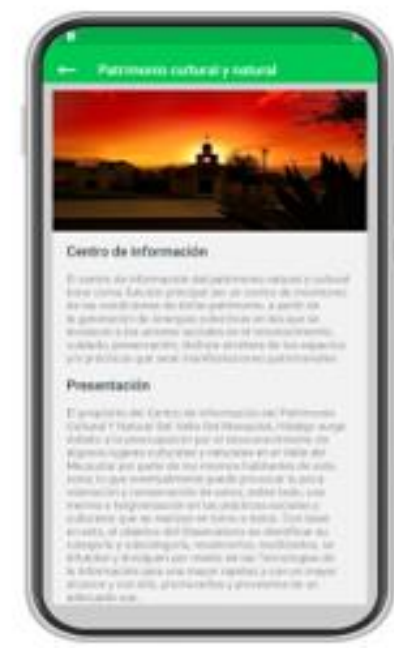

\section{CONCLUSIONES}

Como se mencionó, actualmente existe un sesgo en la información entre los expertos en la conservación del patrimonio y las personas en general; siendo que estas últimas las que están en contacto con los lugares ya sea naturales o culturales, sin embargo, ya que la información no suele acercarse a las personas en general; justamente uno de los objetivos de este Centro de Información es ser el vínculo entre esos dos grandes actores; utilizando dos de las herramientas de las TI como son la aplicación móvil y el sitio web, dado que son herramientas que son, hasta cierto punto asequibles a la sociedad en general. Esto se soporta en los datos mostrados a lo largo del trabajo, sin embargo, también es necesario que además de que se use ese vínculo, es fundamental la identificación de los lugares que pueden ser considerados patrimonio, como también se expuso anteriormente. Por ello, la identificación es la parte en que las TI tienen un rol fundamental, ya que las personas se vuelven partícipes de la selección de lugares por medio de las tecnologías.

Por esta razón, esta fase del proyecto es fundamental, ya que entre más asequible sea la información y sobre todo la interfaz de las TI del Centro de Información, más fácil se utilizará y se retroalimenta.

Con base en esto y los resultados obtenidos, como consecuencia directa de la evaluación se obtuvo un mejoramiento en la interacción del sitio web y de la aplicación móvil, comenzando por el mejoramiento de la calidad de los procesos de desarrollo así como la constante validación de los diferentes módulos que los conforman, así mismo, el manejo de los recursos fue óptimo ya que los cambios fueron radicales pero 
en cuestión de la interfaz ya que el funcionamiento seguía siendo lo que el usuario final esperaba. Así mismo, se identificaron los procesos de riesgo propios del desarrollo con la finalidad de ofrecer al usuario información veraz y así asegurar la confidencialidad, integridad y disponibilidad de los datos que se manejan en las tecnologías.

Con el uso adecuado de la aplicación móvil, se pudo identificar que el apartado "Contacto" permite una actualización y mejora continua de los módulos, ofreciendo al Centro de información la oportunidad de aportar datos útiles que favorezcan en el uso adecuado de estos lugares promoviendo su difusión y mantenimiento adecuados por las organizaciones gubernamentales y no gubernamentales.

Finalmente, destacar que la evaluación de la usabilidad es una parte fundamental del enfoque del desarrollo de software, que permite innovar las funciones y la presentación de cada interfaz para aumentar el conocimiento de los interesados acerca de los problemas de interacción que hayan sido detectados. 


\section{REFERENCIAS}

1. Secretaria de Cultura (SC). (29 de enero de 2018). México es Cultura. Aplicación móvil, 3.7.9. Recuperado el 22 de agosto de 2018, de https://play.google.com/store/apps/details?id=st.conaculta.an droid.activity.

2. Secretaria de Turismo (SECTUR). (08 de diciembre de 2018). Atlas Turístico de México. Aplicación móvil, 1.2. Obtenido de https://play.google.com/store/apps/details?id=com.sectur.atla sturisticomxv2.

3. UNESCO. (1972). Convención sobre la protección del patrimonio mundial, cultural y natural. París, Francia: Organización de las Naciones Unidas para la Educación, la Ciencia y la Cultura.

4. P. P. Cruz, "Sobre el concepto de valor. Una propuesta de integración de diferentes perspectivas", Bordon. Revista Pedagógica., 60 (1), 2008, pp. 99-112. España

5. R. R. Jorge, El imaginario, la memoria y los significados, en la valoración y conservación del patrimonio arquitectónico", Reingtec., vol. 1, 2016, pp. 7-15. México.

6. ICOMOS MÉXICO (2009), “Conclusiones de la Carta de Zacatecas escrita en XXÍX Symposium Internacional de Conservación del Patrimonio Cultural”. Consejo Internacional de Monumentos y Sitios. México, Zacatecas, México.

7. N. M. Raúl, "El proceso de desarrollo de software”, ITCampus Academy 2da edición. 2017.

8. Barroso O. Julio, Cabero A. J. (2013). Las tecnologías de la información y la comunicación aplicadas a la formación y desarrollo curricular. España: Pirámide.

9. Mysql.com. (2019). MySQL: MySQL Workbench. [online] Disponible en: https://www.mysql.com/products/workbench/ [Consultado el 6 Oct. 2019].

10. Mysql.com. (2019). MySQL. [online] Disponible en: https://www.mysql.com/ [Consultado el 6 Oct. 2019].

11. Google Developers. (2019). Overview | Maps JavaScript API | Google Developers. [online] Disponible en: https://developers.google.com/maps/documentation/javascript/tutorial [Consultado el 6 Oct. 2019].

12. Atom. (2019). A hackable text editor for the 21st Century. [online] Disponible en: https://atom.io/docs [Consultado el 6 Oct. 2019].

13. Php.net. (2019). PHP: Documentation. [online] Disponible en: https://www.php.net/docs.php [Consultado el 6 Oct. 2019].

14. Javascript.com. (2019). Learn JavaScript basics with our free JavaScript tutorials for programmers. [online] Disponible en: https://www.javascript.com/about [Consultado el 6 Oct. 2019].

15. MDN. (2019). HTML. [online] Disponible en: https://developer.mozilla.org/es/docs/Web/HTML [Consultado el 6 Oct. 2019]. 
16. R. R. Jorge, "Estudio e identificación del patrimonio cultural y natural en el Valle del Mezquital", Reingtec., vol. 3, 2017, pp. 1-8. México.

17. H. S. Roberto. "Metodología de la investigación. México", 2010 DF.: Mc Graw-Hill.

18. A. A. Enrqiue, "Historia de la Arquitectura Mexicana". (2013) Ciudad de México: Gustavo Gili.

19. M.Onaindia, "Servicios ambientales de los ecosistemas". Amazonía y agua: desarrollo sostenible en el siglo XXI / coord. por Hernando Bernal Zamudio, Carlos Hugo Sierra Hernando, Mario Angulo Tarancón, Miren Onaindia Olalde, 2010, ISBN 978-84-934779-8-1, págs. 29-38.

20. ISO International Standard, ISO 9241-11 (1998): Ergonomic requirements for office work with visual display terminals (VDTs.) - Part 11: Guidance on usability.

21. Solano A. Andrés, F (2015) Evaluación colaborativa de la usabilidad en el desarrollo de sistemas software interactivos. Santiago de Cali. Universidad Autónoma de Occidente. 